\title{
Soy Protein Isolate-Alginate Microspheres for Encapsulation of Enterococcus faecalis HZNU P2
}

\author{
Yu Zhang ${ }^{1}$, Wei Zheng ${ }^{1}$, Jian-Fei Gu ${ }^{1}$, Jian Ni ${ }^{1}$, Ling Wang ${ }^{1}$, Zhen-Xing Tang ${ }^{2}$ and Lu-E \\ Shi $^{1 *}$ \\ ${ }^{1}$ College of Life and Environmental Sciences; Hangzhou Normal University; Hangzhou, Zhejiang - China. ${ }^{2}$ College \\ of Light Industry Science and Engineering; Nanjing Forestry University; Nanjing, Jiangsu - China
}

\begin{abstract}
In this work, the mixture of alginate and soy protein isolate used as a wall material was developed to encapsulate Enterococcus faecalis HZNU P2 (E. faecalis HZNU P2). The survival ability in the simulated gastric fluid (SGF) and bile salt solution, storage stability at different temperatures and release properties in the simulated intestinal fluid (SIF) of encapsulated cells were assessed. The results showed that encapsulation could offer sufficient protection to $\mathrm{E}$. faecalis $H Z N U P 2$. The viability of encapsulated $\mathrm{E}$. faecalis $H Z N U P 2$ did not decrease in SGF at pH 2.5 or 2.0 after $2 \mathrm{~h}$ incubation, while free cells were reduced from 11 to $9.85 \log \mathrm{CFU} / \mathrm{mL}$ in $S G F(p H 2.5)$ at the same exposure time. Only minor viability of encapsulated $\mathrm{E}$. faecalis HZNU P2 lost in 1.0 or $2.0 \%$ bile salt solution for 1 or 2 h exposure, compared with no survival of free E. faecalis HZNU P2 under the same conditions. Encapsulated $\mathrm{E}$. faecalis $H Z N U P 2$ was completely released from the microspheres in SIF within $1 \mathrm{~h}$. The viability of encapsulated $\mathrm{E}$. faecalis $H Z N U P 2$ stored for two weeks at $4{ }^{\circ} \mathrm{C}$ was fully retained. Viabilities of encapsulated $\mathrm{E}$. faecalis HZNU P2, 9.6 and $9.0 \mathrm{Log}$ CFU/g were obtained at 25 and $37^{\circ} \mathrm{C}$ after 21 days storage, respectively. However, around $1.0 \log \mathrm{CFU} / \mathrm{mL}$ of free cells was reduced after two weeks storage at $4^{\circ} \mathrm{C}$. Encapsulated $\mathrm{E}$. faecalis HZNU P2 using soy protein isolate and alginate as wall materials could play an important role in food applications.
\end{abstract}

Key words: Enterococcus faecalis, Encapsulation, Soy protein isolate, Alginate, Stability, Viability

\section{INTRODUCTION}

Probiotics are defined as "live microorganisms which, when administered in adequate amounts, confer a health benefit on the host" (Sohail et al. 2012; Dong et al. 2013; Pan et al. 2013; Shi et al. 2013a). It can provide various health benefits, including preventing diarrhoea, balancing intestinal microflora, stimulating the immune system, improving lactose intolerance, etc. (Rajam et al. 2012; Dong et al. 2013; Khan et al. 2013; Pan et al. 2013; Shi et al. 2013a, b; Chen et al. 2014). However, it has been reported that many factors, such as low $\mathrm{pH}$, hydrogen peroxide, dissolved oxygen, and storage temperature can affect the viability of probiotics (Champagne et al. 2011; Rajam et al. 2012; Dong et al. 2013; Chen et al. 2014). Many ways, such as appropriate selection of acid and bile resistant strains, two-step fermentation, stress adaptation, incorporation of micronutrients and encapsulation, have been proposed to increase the viability of probiotics against the adverse environmental conditions (Anal and Singh 2007; Rajam et al. 2012; Dong et al. 2013; Shi et al. 2013a, b). Among them, encapsulation is one of effective methods that

*Author for correspondence: shilue@126.com 
improve the viability and stability of probiotics and also provide the release of probiotics in the gastrointestinal tract (Krasaekoopt et al. 2003; Dolly et al. 2011; Rajam et al. 2012; Dong et al. 2013; Pan et al. 2013; Shi et al. 2013a, b; Chen et al. 2014). It has many different applications in the food, biomedical, pharmaceutical and cosmetic industries as well as in agriculture and catalysis (Dubey et al. 2009; Dong et al. 2013; Nesterenko et al. 2013).

Alginate, a natural polysaccharide, has been widely used as a wall material for encapsulation. However, encapsulation of the probiotics only using alginate as a wall material can not protect the probiotics effectively from low $\mathrm{pH}$ environment (Krasaekoopt et al. 2003; Rajam et al. 2012; Pan et al. 2013; Shi et al. 2013a, b; Chen et al. 2014). Soy protein isolate can be used as an encapsulation material due to its interesting physico-chemical properties, in particular gelforming and emulsifying properties $(\mathrm{Gu}$ et al. 2009; Dong et al. 2013; Nesterenko et al. 2013). Many works have indicated that relative to sodium caseinate or whey protein, the encapsulated products with soy protein isolate as a wall material exhibit comparable or even better encapsulation efficiency, and higher stability against oxidation (Charve and Reineccius 2009; Rascon et al. 2011; Nesterenko et al. 2013). Generally, soy protein isolate is not only used as an individual coating material, but also can be mixed with polysaccharides (Augustin et al. 2006; Rusli et al. 2006; Nesterenko et al. 2013). However, the application of soy protein isolate in probiotics encapsulation is still very limited.

Enterococci belong to lactic acid bacteria (LAB), which play an important role in the development of the sensory characteristics of fermentation foods, such as sausages and cheeses (Sánchez et al. 2007; Zheng et al. 2015). Some enterococcal strains have been successfully used as preservatives to inhibit the growth of food spoilage microorganisms (Zheng et al. 2015). A new strain, Enterococcus faecalis HZNU P2 from peacock fecal samples was isolated, which showed good tolerance to $\mathrm{NaCl}$ and simulated intestinal juice, good adhesion ability to the intestinal cell, as well as good antimicrobial activity against selected pathogens. However, it did not show good stability in SGF and bile salt solution. Therefore, in present study, it was encapsulated in soy protein isolatealginate microspheres. The tolerance ability to low $\mathrm{pH}$ and bile solution, as well as release characteristic in SIF was investigated. In addition, storage viability of the cells was also determined.

\section{MATERIAL AND METHODS}

\section{The bacterial strain}

The strain E. faecalis HZNU P2 was isolated from the fresh peacock fecal samples, which was collected in China Center for Type Culture Collection (CCTCC). The preservation number is CCTCC M 2014197. The culture was stored at $80^{\circ} \mathrm{C}$ in MRS broth (Oxoid), supplemented with $25 \%$ glycerol. Stock culture was propagated in MRS broth at $37^{\circ} \mathrm{C}$ for $24 \mathrm{~h}$ and cell biomass was harvested after centrifugation at $3000 \mathrm{~g}, 4^{\circ} \mathrm{C}$ for 10 min, washed by sterile water, and then resuspended in saline solution $(0.85 \%)$. The cell suspension was used for the following work.

\section{Encapsulation of $E$. faecalis HZNU P2 in soy protein isolate-alginate microspheres}

Soy protein isolate (Yunxin Cooperation Inc., Shandong, China; protein concentration $>90 \%$, fat concentration $<1.0 \%$, moisture content $<7.0 \%$ ) and sodium alginate (low viscosity, Sigma Aldrich, Shanghai) were sterilized at 110 and $121^{\circ} \mathrm{C}$ for $15 \mathrm{~min}$, respectively. Soy protein isolate-alginate microspheres were prepared according to previous works (Pan et al. 2013; Shi et al. 2013a, b; Chen et al. 2014; Tang et al. 2013, 2015). Briefly, E. faecalis HZNU P2 was mixed with sodium alginate and soy protein isolate. The mixture (alginate/soy protein isolate $=1 / 3, \mathrm{v} / \mathrm{v}$, alginate concentration 1.0\%) was injected through an Inotech Encapsulator IER-50 (Inotech Biosystems Intl. Inc., Reppischhof, Switzerland) into $100 \mathrm{mM} \mathrm{CaCl}{ }_{2}$ at gently stirring $(100 \mathrm{rpm})$ using $450 \mu \mathrm{m}$ nozzle. The microspheres were allowed to stand for $30 \mathrm{~min}$ for gelification, then washed by distilled water, and subsequently kept in sterile conical tubes (High Clarity Polypropylene, Fisher Scientific Inc., Shanghai, China).

The encapsulation yield of E. faecalis HZNU P2 was calculated as follows: encapsulate yield $(\%)=$ (quantity of $E$. faecalis HZNU P2 released from the broken microspheres/quantity of E. faecalis HZNU P2 initially taken to prepare the microspheres $) \times 100$. The size of microspheres was measured using an optical microscope (Carl Zeiss, Germany). Fifty microspheres were randomly picked to determine the size. The size of 
each sample was presented as the mean size \pm standard deviation (SD).

\section{Bacterial enumeration}

The microspheres containing E. faecalis HZNU P2 $(0.50 \mathrm{~g})$ were broken in $4.5 \mathrm{~mL}, 50 \mathrm{mM}$ sterile sodium citrate solution by gently shaking at room temperature. Appropriate dilutions were made in $0.85 \%$ saline solution. Diluted solutions $(100 \mu \mathrm{L})$ were plated onto MRS agar. Colonies of $E$. faecalis HZNU P2 were enumerated after the incubation at $37^{\circ} \mathrm{C}$ for $24 \mathrm{~h}$. Free E. faecalis HZNU P2 was 10 times serially diluted with saline solution and $100 \mu \mathrm{l}$ aliquots were plated on MRS agar. Colonies of E. faecalis HZNU P2 were enumerated according to the method used for enumeration of encapsulated E. faecalis HZNU P2.

\section{Resistance ability of free and encapsulated $E$. faecalis HZNU P2 to SGF}

The tolerance of free and encapsulated E. faecalis HZNU P2 to the simulated gastric fluid (SGF, 0.20 $\% \mathrm{NaCl}$ ) was determined as described previously (Pan et al. 2013; Shi et al. 2013a, b; Tang et al. 2013, 2015; Chen et al. 2014). SGF was adjusted to $\mathrm{pH} 2.0$ and 2.5 with concentrated $\mathrm{HCl}$. A 0.50 $\mathrm{mL}$ cell suspension or $0.50 \mathrm{~g}$ encapsulated $E$. faecalis HZNU P2 was mixed with $4.5 \mathrm{~mL}$ SGF and incubated at $37^{\circ} \mathrm{C}$ for $10,30,60,90$ and 120 min. After the specified time intervals, samples were harvested and immediately used for the enumeration of viable cells according to the method described above.

\section{Survival of free and encapsulated $E$. faecalis} HZNU P2 in bile salt solution

Tolerance of the free and encapsulated E. faecalis HZNU P2 to bile salt solutions with various concentrations were carried out as described previously (Pan et al. 2013; Shi et al. 2013a, b; Chen et al. 2014; Tang et al. 2013, 2015). Briefly, $0.50 \mathrm{~g}$ microspheres or $0.50 \mathrm{~mL}$ cell suspension was transferred in test tubes containing $4.5 \mathrm{~mL}$, 1.0 or $2.0 \%$ bile salt solution (Sigma-Aldrich, Shanghai) and incubated at $37^{\circ} \mathrm{C}$ for 1 or $2 \mathrm{~h}$. The enumeration of viable cells was carried out according to the method described above.

\section{Release of encapsulated $E$. faecalis HZNU P2 in SIF}

The release of encapsulated E. faecalis HZNU P2 in SIF ( $\mathrm{pH} 6.8,50 \mathrm{mM} \mathrm{KH} \mathrm{PO}_{4}$ ) was tested using the method described previously (Pan et al. 2013; Shi et al. 2013a, b; Chen et al. 2014; Tang et al. 2013, 2015). Microspheres of $0.50 \mathrm{~g}$ were added to $4.5 \mathrm{~mL} \mathrm{SIF}$ and the mixture was incubated at $37^{\circ} \mathrm{C}$. A $100 \mu \mathrm{L}$ the supernatant was taken at time intervals of $0,10,30,60,90$ and $120 \mathrm{~min}$ and then serially diluted in $0.85 \%$ saline solution. The same volume of the fresh medium was added to replace the volume of the withdrawn samples. The enumeration of viable cells was carried out according to the method described above.

\section{Shelf lives of free and encapsulated $E$. faecalis HZNU P2}

The survival of free and encapsulated E. faecalis HZNU P2 were tested at 4,25 and $37^{\circ} \mathrm{C}$. The samples were taken at time intervals of storage and the number of viable cells was determined as the method described above.

\section{Statistics analysis}

All the experiments were conducted in triplicate and the results were expressed as mean value \pm standard deviation (SD). The data was analyzed using Origin 8.0 for Windows. The significant differences were compared using Student's t test. The statistical significance was accepted at the level of $\mathrm{P}<0.05$.

\section{RESULTS AND DISCUSSION}

One of necessary pre-requisites for encapsulation method is high encapsulation yield (Chen et al. 2014). The initial numbers in E. faecalis HZNU $\mathrm{P} 2$ suspension were about $11 \log \mathrm{CFU} / \mathrm{mL}$. The numbers in microspheres were $10.98 \mathrm{log} \mathrm{CFU} / \mathrm{g}$. High encapsulation rate $(\approx 100 \%)$ was achieved. Therefore, soy protein isolate-alginate matrices had a good compatibility with E. faecalis HZNU P2.

The diameters of soy protein isolate-alginate microspheres obtained in this work were around $800 \pm 10 \mu \mathrm{m}$ (data not shown). The size of the microspheres is an important factor for the application in food industry. Large microspheres can adversely affect the texture of the foods; small microspheres may not provide sufficient protection to probiotics (Shi et al. 2013a, b; Chen et al. 2014). In this study, soy protein isolate-alginate microspheres were below $1.0 \mathrm{~mm}$. It would not produce enough detrimental effect on the texture of foods. 
Stability of free and encapsulated $E$. faecalis HZNU P2 in SGF

One of major problems for the probiotic foods is the low survival of the cells in the gastric $\mathrm{pH}$ (Pan et al. 2013; Shi et al. 2013a, b; Chen et al. 2014). Free and encapsulated E. faecalis HZNU P2 were tested for the survival ability in SGF (pH 2.0 and 2.5) (Fig. 1). Evidently encapsulation improved the stability of E. faecalis HZNU P2 in SGF. The numbers of encapsulated E. faecalis HZNU P2 did not decrease after $2.0 \mathrm{~h}$ incubation in SGF $(\mathrm{pH} 2.5$ and 2.0). However, the viability of free E. faecalis HZNU P2 decreased from around $11 \mathrm{Log}$ CFU/mL to 9.85 and $9.45 \mathrm{Log} \mathrm{CFU} / \mathrm{mL}$ for $2.0 \mathrm{~h}$ exposure at SGF pH 2.5 and 2.0, respectively. Many studies have indicated that encapsulation can help in improving the survival of the cells in SGF (Chandramouli et al. 2004; Krasaekoopt et al. 2004; Iyer and Kailasapathy 2005; Annan et al. 2008; Chavarri et al. 2010; Pan et al. 2013; Shi et al. 2013a, b; Chen et al. 2014; Sathyabama et al. 2014). To date, the use of plant protein isolate as a wall material for probiotic encapsulation, particularly soy protein isolate, is extremely limited. Klemmer et al. (2011) reported that the viable cell numbers in pea protein isolate-alginate capsules containing $B$. adolescentis were reduced $1.0 \mathrm{Log} \mathrm{CFU} / \mathrm{g}$ over a $2 \mathrm{~h}$ exposure in SGF at $\mathrm{pH}$ 2.0 and $37^{\circ} \mathrm{C}$. Kotikalapudi et al. (2010) found that encapsulated Lactobacillus acidophilus in pea protein isolate-alginate capsules showed high resistance to $\mathrm{SGF}$ at $\mathrm{pH} 2.0$.

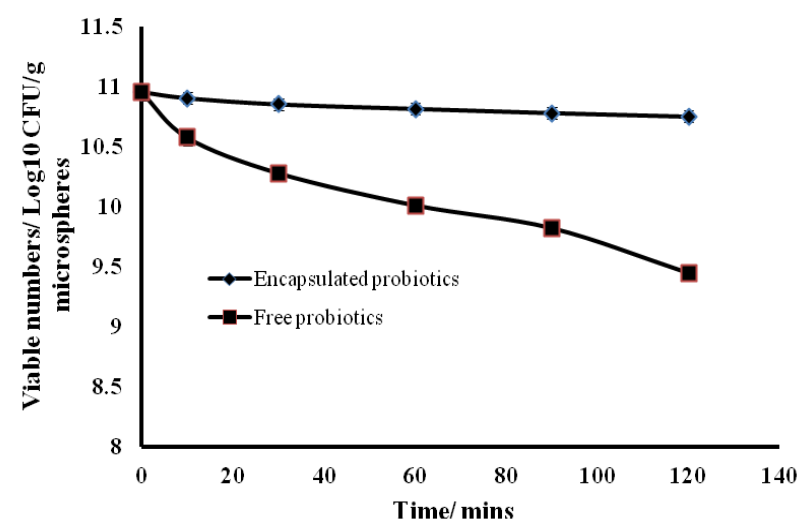

Figure 1 - pH stability of free and encapsulated E. faecalis HZNU P2 in simulated gastric fluid (SGF) pH 2.0 and 2.5. Fig. 1-A The stability of free and encapsulated E. faecalis HZNU P2 in SGF $\mathrm{pH}$ 2.5. Fig. 1-B The stability of free and encapsulated E. faecalis HZNU P2 in SGF $\mathrm{pH} 2.0$.

\section{Stability of free and encapsulated $E$. faecalis HZNU P2 in bile salt solution}

The stability results of free and encapsulated $E$. faecalis HZNU P2 subjected to bile salt solutions (1.0 and 2.0\%) are shown in Table 1. No survival of free E. faecalis HZNU P2 cells was found after $1 \mathrm{~h}$ exposure in 1.0 or $2.0 \%$ bile salt solution. The sensitivity of many strains of probiotics to bile salt solutions has been reported by many researchers (Amor et al. 2002; Picot and Lacroix 2004; Pan et al. 2013; Shi et al. 2013a, b; Chen et al. 2014; Sathyabama et al. 2014). Table 1 shows slight reductions of encapsulated E. faecalis HZNU P2 after $2 \mathrm{~h}$ incubation in 1.0 or $2.0 \%$ bile salt solutions. Many studies have shown that encapsulation can improve the survival numbers of probiotics in bile salt conditions (Krasaekoopt et al. 2003; Chandramouli et al. 2004; Iyer and Kailasapathy 2005; Mandal et al. 2006; Pan et al. 2013; Shi et al. 2013a, b; Chen et al. 2014; Sathyabama et al. 2014). However, in contrast to the present results, Trindade and Grosso (2000) observed that encapsulation of Bifidobacterium bifidum and L. acidophilus in alginate beads was not effective in protecting the cells from 2.0 to $4.0 \%$ bile salt solutions. In the present study, high survival numbers of E. faecalis HZNU P2 in soy protein isolate-alginate microspheres after bile salt solution treatment was pre-requisite for providing a beneficial health effect to the host. 
Table 1 - Survival of free and encapsulated E. faecalis HZNU P2 after treatment in bile salt solutions of 1.0 and $2.0 \%$ for 1 and $2 \mathrm{~h}$ ( $\mathrm{Log} \mathrm{CFU} / \mathrm{mL}$ or $\mathrm{g}$ microspheres)

\begin{tabular}{ccccc}
\hline $\begin{array}{c}\text { Incubation } \\
\text { time (h) }\end{array}$ & Sample & \multicolumn{3}{c}{ Bile concentration (\%) } \\
& & 0 & 1 & 2 \\
\hline \multirow{4}{*}{1} & Free & $10.944 \pm$ & 0 & 0 \\
& & 0.12 & & \\
& Encapsulated & $10.854 \pm$ & $10.645 \pm$ & $10.402 \pm$ \\
& & 0.07 & 0.09 & 0.13 \\
& Free & $10.954 \pm$ & 0 & 0 \\
2 & & 0.11 & & \\
& Encapsulated & $10.944 \pm$ & $10.406 \pm$ & $10.277 \pm$ \\
& & 0.09 & 0.12 & 0.14 \\
\hline
\end{tabular}

\section{Release of encapsulated E. faecalis HZNU P2 in SIF}

The release of probiotics from the microspheres in intestinal tract is essential for the growth and colonization of probiotics (Pan et al. 2013; Shi et al. 2013a, b; Chen et al. 2014). Figure 2 shows the release of encapsulated E. faecalis HZNU P2 in SIF. When encapsulated E. faecalis HZNU P2 was placed into SIF, the microspheres began to swell and eventually disintegrated. E. faecalis HZNU P2 could be released from the soy protein isolatealginate microspheres in SIF within $1 \mathrm{~h}$. Analogous probiotic release behaviour was reported by Klemmer et al. (2011) for encapsulated $B$. adolescentis in pea protein isolate-alginate. It is suggested that the developed encapsulated formulations have potential for the targeted delivery of probiotics to the lower gastrointestinal tract. Chen et al. (2014) found that $70 \%$ of encapsulated L. bulgaricus in whey protein isolate-alginate microspheres could be released in SIF within $1 \mathrm{~h}$. Tang et al. (2015) clearly demonstrated that encapsulated phage $\mathrm{K}$ in whey protein-alginate microspheres had a burst release

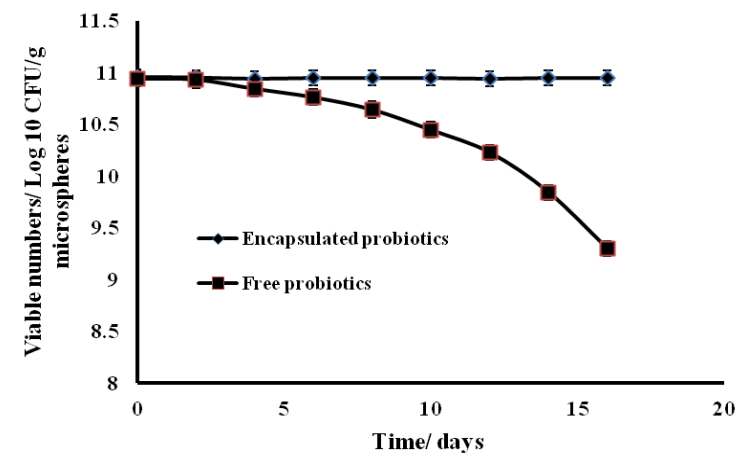

in the first $1 \mathrm{~h}$, followed by a sustained release for up to $2 \mathrm{~h}$.

\section{Storage stability of free and encapsulated $E$. faecalis HZNU P2}

Storage stability of the free and encapsulated $E$. faecalis $\mathrm{HZNU} \mathrm{P} 2$ at 4,25 and $37^{\circ} \mathrm{C}$ is shown in Figure 3 . The viability of encapsulated $E$. faecalis HZNU P2 showed higher storage stability compared to that of free E. faecalis HZNU P2. Viability of the free E. faecalis HZNU P2 was reduced from 10.94 to $10.76 \log \mathrm{CFU} / \mathrm{mL}$ after one week, and around $9.80 \log \mathrm{CFU} / \mathrm{mL}$ after two weeks (Fig. 3A). However, the numbers of encapsulated E. faecalis HZNU P2 were only slightly reduced (Fig. 3A). The survival numbers of encapsulated cells were reduced from around 11.0 Log CFU/g to 9.6 and 9.0 Log CFU/g after 21 days storage at 25 and $37{ }^{\circ} \mathrm{C}$, respectively (Fig. 3B). Therefore, soy protein isolate-alginate microspheres could improve the storage stability of E. faecalis HZNU P2. Several studies have indicated that encapsulated probiotics in alginatebased microspheres had better storage ability than free cells (Krasaekoopt et al. 2003; Yew et al. 2011; Pan et al. 2013; Shi et al. 2013a, b; Chen et al. 2014).

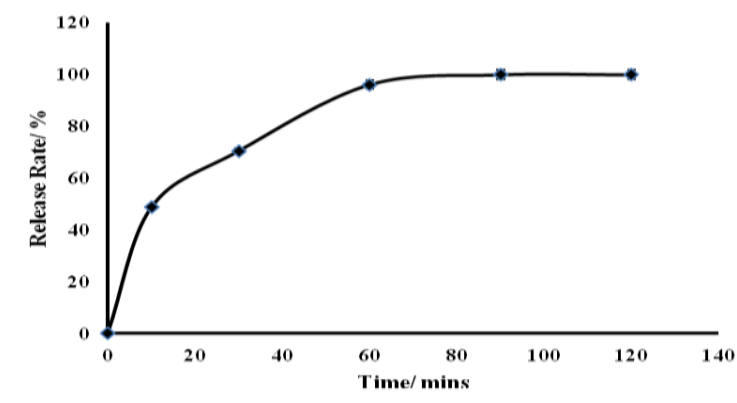

Figure 2 - Release of encapsulated E. faecalis HZNU $\mathrm{P} 2$ in simulated intestine fluid (SIF).

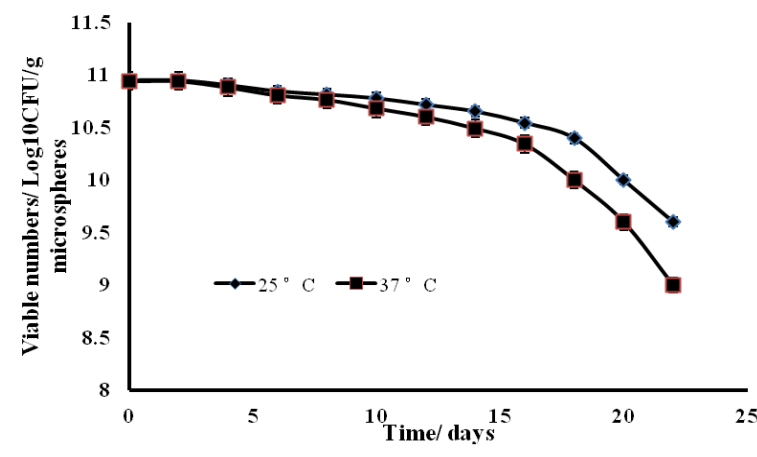

Figure 3 - Storage stability of free and encapsulated E. faecalis HZNU P2 at 4, 25 and $37^{\circ} \mathrm{C}$. Fig. 3-A Storage stability of free and encapsulated E. faecalis $\mathrm{HZNU} \mathrm{P} 2$ at $4^{\circ} \mathrm{C}$. Fig. 3-B Storage stability of encapsulated E. faecalis $\mathrm{HZNU} \mathrm{P} 2$ at 25 and $37^{\circ} \mathrm{C}$. 


\section{CONCLUSIONS}

This study demonstrated that E. faecalis HZNU P2 could be encapsulated in soy protein isolatealginate microspheres. Microspheres provided good protection to E. faecalis HZNU P2 against adverse influences of gastric and bile salt conditions. The encapsulated cells showed a rapid release in SIF. The results suggested that soy protein isolate-alginate microspheres could be a potential candidate for oral delivery of $E$. faecalis HZNU P2 and could pave the way for utilizing the market-friendly plant-based protein for producing controlled delivery systems for the probiotic industry.

\section{ACKNOWLEDGEMENTS}

This study was financially supported by the Xinmiao Talent Program of Zhejiang Province (2013R421006, 2012R421003).

\section{REFERENCES}

Amor KB, Breeuwer P, Rombouts P, Verbaarschot FM, Akkermans ADL, De Vos W, et al. Multiparametric flow cytometry and cell sorting for the assessment of viable, injured and dead bifidobacterium cells during bile salt stress. Appl Environ Microbiol. 2002; 68: 5209-5216.

Anal AK, Singh H. Recent advances in microencapsulation of probiotics for industrial applications and targeted delivery. Trends Food Sci Tech. 2007; 18: 240-251.

Annan NT, Borza AD, Truelstrup Hansen L. Encapsulation in alginate-coated gelatin microspheres improves survival of the probiotic Bifidobacterium Adolescentis $15703 \mathrm{~T}$ during exposure to simulated gastro-intestinal conditions. Food Res Inter. 2008; 41: 184-193.

Augustin MA, Sanguansri L, Bode O. Maillard reaction products as encapsulants for fish oil powders. J Food Sci. 2006; 71: 25-32.

Champagne CP, Gardner NJ, Roy D. Challenges in the addition of probiotic cultures to foods. Crit Rev Food Sci Nutr. 2005; 45: 61-84.

Champagne CP, Ross RP, Saarela M, Hansen KF, Charalampopoulos D. Recommendations for the viability assessment of probiotics as concentrated cultures and in food matrices. Inter J Food Microbiol. 2011; 149: 185-193.
Chandramouli V, Kailasapathy K, Peiris P, Jones M. An improved method of microencapsulation and its evaluation to protect Lactobacillus spp in simulated gastric conditions. J Microbiol Meth. 2004; 57: 27 35 .

Charve J, Reineccius GA. Encapsulation performance of proteins and traditional materials for spray dried flavors. J Agri Food Chem. 2009; 57: 2486-2492.

Chavarri M, Maranon I, Ares R, Ibanes FC, Marzo F, Villaran Mdel C. Microencapsulation of a probiotic and prebiotic in alginate-chitosan capsules improves survival in simulated gastro-intestinal conditions. Inter J Food Microbiol. 2010; 142: 185-189.

Chen MY, Zheng W, Dong QY, Li ZH, Shi LE, Tang ZX. Activity of encapsulated Lactobacillus bulgaricus in alginate-whey protein microspheres. Braz Arch Biol Tech. 2014; 57: 736-741.

Dolly P, Anishaparvin A, Joseph GS, Anandharamakrishnan C. Microencapsulation of Lactobacillus plantarum (mtcc 5422) by sprayfreeze-drying method and evaluation of survival in simulated gastrointestinal conditions. $J$ Microencapsul. 2011; 28: 568--574.

Dong QY, Chen MY, Xin Y, Qin XY, Cheng Z, Shi LE, et al. Alginate-based and protein-based materials for probiotics encapsulation: A review. Inter J Food Sci Technol. 2013; 48: 1339-1351.

Dubey R, Shami TC, Bhasker Rao KU. Microencapsulation technology and application. Defence Sci J.2009; 59: 82-95.

Gu X, Campbell LJ, Euston SR. Effects of different oils on the properties of soy protein isolate emulsions and gels. Food Res Inter. 2009; 42: 925-932.

Iyer C, Kailasapathy K. Effect of co-encapsulation of probiotics with prebiotics on increasing the viability of encapsulated bacteria under in vitro acidic and bile salt conditions and in yogurt. J Food Sci. 2005; 70: 18-23.

Khan NH, Korber DR, Low NH, Nickerson MT. Development of extrusion-based legume protein isolate-alginate capsules for the protection and delivery of the acid sensitive probiotic, Bifidobacterium adolescentis. Food Res Inter. 2013; 54: 730-737.

Klemmer KJ, Korber DR, Low NH, Nickerson MT. Pea protein-based capsules for probiotic and prebiotic delivery. Inter J Food Sci Technol. 2011; 46: 22482256.

Kotikalapudi BL, Low NH, Nickerson MT, Korber DR. In vitro characterization of probiotic survival, adherence and antimicrobial resistance: Candidate selection for encapsulation in a pea protein isolatealginate delivery system. Inter J Pro Pre. 2010; 5: 112 . 
Krasaekoopt W, Bhandari B, Deeth, H. Evaluation of encapsulation techniques of probiotics for yoghurt. Inter Dairy J. 2003; 13: 3-13.

Krasaekoopt W, Bhandari B, Deeth H. The influence of coating materials on some properties of alginate beads and survivability of microencapsulated probiotic bacteria. Inter Dairy J. 2004; 14: 737-743.

Mandal S, Puniya AK, Singh K. Effect of alginate concentrations on survival of microencapsulated Lactobacillus casei NCDC-298. Inter Dairy J. 2006; 16: 1190-1195.

Nesterenko A, Alric I, Silvestre F, Durrieu V. Vegetable proteins in microencapsulation: a review of recent interventions and their effectiveness. Ind Crop Prod. 2013; 42: 469-479.

Pan LX, Fang XJ, Yu Z, Xin Y, Liu XY, Shi L E, et al. Encapsulation in alginate-skim microspheres improves viability of Lactobacillus bulgaricus in stimulated gastro-intestinal conditions. Inter J Food Sci Nutr. 2013; 64: 380-384.

Picot A, Lacroix C. Encapsulation of bifidobacteria in whey protein-based microcapsules and survival in simulated gastrointestinal conditions and in yoghurt. Inter Dairy J. 2004; 14: 505-515.

Rajam R, Karthik P, Parthasarathi S, Joseph GS, Anandharamakrishnan C. Effect of whey proteinalginate wall systems on survival of microencapsulated Lactobacillus plantarum in simulated gastrointestinal conditions. J Funct Foods. 2012; 4: 891-898.

Rascon MP, Beristain CI, Garcie HS, Salgado MA. Carotenoid retention and storage stability of spraydried paprika oleoresin using gum Arabic and soy protein isolate as wall materials. Food Sci Technol. 2010; 44: 549-557.

Rusli JK, Sanguansri L, Augustin MA. Stabilization of oils by microencapsulation with heated proteinglucose syrup mixtures. J Am Oil Chem Soc. 2006; 83: 965-971.

Sánchez, J, Basanta, A, Gómez-Sala, B, Herranz, C, Cintas, LM, Hernández, PE. Antimicrobial and safety aspects, and biotechnological potential of bacteriocinogenic enterococci isolated from mallard ducks (Anas platyrhynchos). Inter J Food Microbiol. 2007; 117: 295-305.
Sathyabama S, Kumer MR, Devi PB, Vijayabharathi R, Priyadharisini VB. Co-encapsulation of probiotics with prebiotics on alginate matrix and its effect on viability in simulated gastric environment. LWT-Food Sci Technol. 2014; 57: 419-425.

Shi LE, Li ZH, Li DT, Xu M, Chen HY, Zhang ZL, et al. Encapsulation of probiotics Lactobacillus bulgaricus in alginate-milk microspheres and evaluation of survival in simulated gastrointestinal conditions. J Food Eng. 2013a; 117: 99-104.

Shi LE, Zhang ZL, Song YQ, Zhou ML, Yu WM, Tang Z X. Encapsulation of Lactobacillus bulgaricus in carragenan-locust bean gum coated milk microspheres with double layer structure. LWT-Food Sci Technol. 2013b; 54: 147-151.

Sohail A, Turner MS, Prabawati EK, Coombes AGA, Bhandari B. Evaluation of Lactobacillus rhamnosus GG and Lactobacillus acidophilus NCFM encapsulated using a novel impinging aerosol method in fruit food products. Inter J Food Microbiol. 2012; 157: $162-166$.

Tang ZX, Huang XQ, Baxi S, Chambres JR, Sabour PM, Wang Q. Whey protein improves survival and release characteristics of bacteriophage Felix O1 encapsulated in alginate microspheres. Food Res Inter. 2013; 52: 460-466.

Tang ZX, Huang XQ, Sabour PM, Chambers JR, Wang Q. Preparation and characterization of dry powder bacteriophage $\mathrm{K}$ for intestinal delivery through oral administration. LWT-Food Sci Technol. 2015; 60: 263-270.

Trindade CSF, Grosso CRF. The effect of the immobilization of Lactobacillus acidophilus and Bifidobacterium lactis in alginate on their tolerance to gastrointestinal secretions. Milchwissenschaft-Milk Sci Inter. 2000; 55: 496-499.

Yew SE, Lim TJ, Lew LC, Bhat R, Mat-Easa A, Liong MZ. Development of a probiotic delivery system from agrowastes, soy protein isolate, and microbial transglutaminase. J Food Sci. 2011; 76: H108-H115.

Zheng, W, Zhang, Y, Lu, HM, Li, DT, Zhang, ZL, Tang, ZX, et al. Antimicrobial activity and safety evaluation of Enterococcus faecium KQ 2.6 isolated from peacock feces. BMC Biotechnol. 2015; 15: 30. 